\title{
Exact Solutions of Scalar Bosons in the Presence of the Aharonov-Bohm and Coulomb Potentials in the Gravitational Field of Topological Defects
}

\author{
Abdelmalek Boumali $(\mathbb{D})$ and Houcine Aounallah \\ Laboratoire de Physique Appliquée et Théorique, Université Larbi Tébessi-Tébessa, 12000 Tébessa, Algeria \\ Correspondence should be addressed to Abdelmalek Boumali; boumali.abdelmalek@gmail.com
}

Received 18 November 2017; Revised 21 January 2018; Accepted 19 February 2018; Published 1 April 2018

Academic Editor: Won Sang Chung

Copyright ( 12018 Abdelmalek Boumali and Houcine Aounallah. This is an open access article distributed under the Creative Commons Attribution License, which permits unrestricted use, distribution, and reproduction in any medium, provided the original work is properly cited. The publication of this article was funded by $\mathrm{SCOAP}^{3}$.

\begin{abstract}
We analyze the relativistic quantum motion of a charged scalar particles in the presence of an Aharonov-Bohm and Coulomb potentials in the space-times produced by an idealized cosmic string and global monopole. We have calculated and discussed the eigensolutions of DKP equation and their dependence on both the geometry of the space-times and coupling constants parameters.
\end{abstract}

\section{Introduction}

The analysis of gravitational interactions with a quantum mechanical system has recently attracted attention in particle physics and has been an active field of research. The general way to understand the interaction between relativistic quantum mechanical particles and gravity is to solve the general relativistic form of their wave equations. These solutions are valuable tools for examining and improving models and numerical methods for solving complicated physical problems.

The DKP equation is a relativistic wave equation which describes spin 0 and spin 1 particles, and it is a direct generalization to the Dirac particles of integer spin in which one replaces the gamma matrices by beta metrics, where the four beta matrices satisfy the Kemmer algebra [1-4]. One important question related to the DKP equation concerns the equivalence between its spin 0 and 1 sectors and the theories based on the second-order KG and Proca equations. The Dirac-like DKP equation is not new and dates back to the 1930s. Historically, the loss of interest in the DKP equation stems from the equivalence of the DKP approach to the KG and Proca descriptions in on-shell situations, in addition to the greater algebraic complexity of the DKP formulation. However, in the 1970s, this supposed equivalence was questioned in several situations involving the breaking of symmetries and the hadronic process, showing, that in some cases, the DKP and KG theories can give different results. Moreover, the DKP equation appears to be richer than the KG equation if interactions are introduced.

The topological defects play an important role in physical properties of systems, and they appear in gravitation as monopoles, strings, and walls. Among them, cosmic string and monopole seem to be the best candidates to be observed. Cosmic strings and global monopoles are exotic topological objects: they do not produce local gravitational interaction; however, they modify the geometry of the space-time, producing planar and solid angle deficit, respectively [5].

The effect of the Aharonov-Bohm potential (AB) is a purely quantum phenomenon which has no classical equivalent. The eminent work of AB in 1959 revealed that the electromagnetic potentials are in reality fundamental physical objects that can manifest themselves in the matter where the corresponding fields exert no force (Lorentz's force is zero) [6]. They proposed an experiment to test, in quantum mechanics, the coupling of electric charges to electromagnetic field strengths through a local interaction with the electromagnetic potential $\mathbf{A}$, but not with the field strengths themselves. Also, they predicted a quantum interference effect due to the motion of charged particles in 
regions where $\mathbf{B}(\mathbf{E})$ vanishes, but not $\mathbf{A}$, leading to a nonlocal gauge-invariant effect depending on the flux of the magnetic field in the inaccessible region, in the magnetic case, and on the difference of the integrals over time of time-varying potentials, in the electric case [7].

The eigenproblem of the Kinetic Angular Momentum (KAM) of the electron in the Aharonov-Bohm effect has been solved by Kretzschmar [8]. The total Hilbert space of the eigenfunctions is split into two subspaces and the symmetry of the motion of the electron around the magnetic flux makes Pauli's criterion inapplicable [9-12]: that means the condition of admissibility of the wave function in the region of $A B$ potential. In this way, Henneberger [9], based on the Pauli criterion, conclude that (i) the single valuedness imposed by Aharonov and Bohm is not the correct one and, consequently, (ii) the $\mathbf{A B}$ effect, if it exists, may not be interpreted as scattering. Roy and Singh [10] and $\mathrm{Li}[11,12]$ showed that the Pauli criterion cannot be applied in the time-dependent case: we note here that the inapplicability of the Pauli criterion means that (i) the KAM does not satisfy the fundamental commutation relations of the angular momentum and (ii) it reflects the breakdown of the symmetry of the electron's motion around the solenoid. Thus, as argued by $\mathrm{Li}[11,12]$, in the presence of $\mathbf{A B}$ potential, the KAM of the electron does not satisfy the fundamental commutation relations of the angular momentum even when the electron is restricted to the doubly connected space where it does not touch the magnetic field on the $z$-axis: the region where the magnetic field exists and is inaccessible to the electron should also be taken into account in the physically meaningful commutation relations. This situation has been used in the case of the interaction of the spin 0 particle with an $\mathbf{A B}$ potential of two and three dimensions by the application of the Duffin-Kemmer-Petiau equation in Minkowski spacetime [13].

The principal aim of this paper is to solve the DKP equation in the presence of a Coulomb and $\mathbf{A B}$ potentials in the gravitational field of topological defects. In the presence of AB potential, we will show that the admissibility of the wave function is inapplicable [8-12]. The inapplicability of the Pauli criterion reflects the breakdown of the symmetry of the particle's motion around the solenoid [11, 12].

The outline of this paper is as follows. In Section 2, a generalized DKP equation is introduced in a curved spacetime. In Section 3, the eigenfunctions of scalar bosons in the presence of the Aharonov-Bohm and Coulomb potentials in the gravitational field of topological defects such as cosmic string and global monopole have been discussed in Sections 3 and 4 . Finally, Section 5 will be a conclusion.

\section{The DKP Equation in Curved Space}

2.1. The Formalism. The first-order relativistic DKP equation for a free charged scalar bosons of mass $M$ in flat space-time is given by [1-4]

$$
\left(i \beta^{\mu} \partial_{\mu}-\frac{M c}{\hbar}\right) \psi=0
$$

where $\beta^{\mu}(\mu=0,1,2,3)$ are the DKP matrices which satisfy the following commutation rules:

$$
\beta^{\kappa} \beta^{v} \beta^{\lambda}+\beta^{\lambda} \beta^{v} \beta^{\kappa}=g^{\kappa \nu} \beta^{\lambda}+g^{\nu \lambda} \beta^{\kappa},
$$

and $g^{\mu \nu}=\operatorname{diag}(1,-1,-1,-1)$ is the Minkowski metric tensor. For the flat space, the beta matrices are chosen as follows [14]:

$$
\begin{aligned}
& \beta^{0}=\left(\begin{array}{cc}
\nu & 0 \\
\widetilde{0}_{T} & \mathbf{0}
\end{array}\right), \\
& \beta^{i}=\left(\begin{array}{cc}
\widehat{0} & \rho^{i} \\
-\rho_{T}^{i} & \mathbf{0}
\end{array}\right),
\end{aligned}
$$

with $\widehat{0}, \widetilde{0}, \mathbf{0}$ as $2 \times 2,2 \times 3,3 \times 3$ zero matrices, respectively, and

$$
\begin{aligned}
\nu & =\left(\begin{array}{ll}
0 & 1 \\
1 & 0
\end{array}\right), \\
\rho^{1} & =\left(\begin{array}{ccc}
-1 & 0 & 0 \\
0 & 0 & 0
\end{array}\right), \\
\rho^{2} & =\left(\begin{array}{ccc}
0 & -1 & 0 \\
0 & 0 & 0
\end{array}\right), \\
\rho^{3} & =\left(\begin{array}{ccc}
0 & 0 & -1 \\
0 & 0 & 0
\end{array}\right) .
\end{aligned}
$$

In curved space-time, (1) is written by [15-17]

$$
\left\{i \widetilde{\beta}^{\mu}\left(\partial_{\mu}+\frac{1}{2} \omega_{\mu a b} S^{a b}-\frac{i e}{\hbar c} A_{\mu}\right)-\frac{M c}{\hbar}\right\} \Psi=0,
$$

where $A_{\mu}$ denotes the vector potential associated with the electromagnetic field and $S^{a b}=\left[\beta^{a}, \beta^{b}\right]$ and $\widetilde{\beta}^{\mu}$ are the Kemmer matrices in curved space-time. These matrices are related to their Minkowski counterparts, $\beta$, via

$$
\widetilde{\beta}^{\mu}=e_{(a)}^{\mu} \beta^{a}
$$

The following tetrad relations and the spin connection are calculated by using the relation,

$$
\omega_{\mu a b}=e_{I(a) l} e_{(b)}^{j} \Gamma_{j \mu}^{l}-e_{(b)}^{j} \partial_{\mu} e_{I(a) j},
$$

where

$$
\Gamma_{\nu \lambda}^{\mu}=\frac{g^{\mu \rho}}{2}\left(g_{\rho v, \lambda}+g_{\rho \lambda, \nu}-g_{\nu \lambda, \rho}\right)
$$

are the Christoffel symbols [18].

Now, with the aids of (6), (7), and (8), spin connection coefficients are as follows.

(i) For the metric corresponding to a cosmic string with [19-22]

$$
d s^{2}=d t^{2}-d r^{2}-r^{2} d \theta^{2}-a^{\prime 2} r^{2} \sin ^{2} \theta d \varphi^{2},
$$


where $-\infty<t<+\infty, 0 \leqslant r, 0 \leqslant \theta \leqslant \pi$, and $0 \leqslant \varphi \leqslant$ $2 \pi, \omega_{\mu a b}$ are

$\omega_{\theta a b}=\left(\begin{array}{cccc}0 & 0 & 0 & 0 \\ 0 & 0 & 1 & 0 \\ 0 & -1 & 0 & 0 \\ 0 & 0 & 0 & 0\end{array}\right)$

$\omega_{\varphi a b}=\left(\begin{array}{cccc}0 & 0 & 0 & 0 \\ 0 & 0 & 0 & a^{\prime} \sin \theta \\ 0 & 0 & 0 & a^{\prime} \cos \theta \\ -a^{\prime} \sin \theta & -a^{\prime} \cos \theta & 0 & 0\end{array}\right)$.

Here the tetrad $e_{(a)}^{\mu}$ are chosen to be

$$
e_{(a)}^{\mu}=\left(\begin{array}{cccc}
1 & 0 & 0 & 0 \\
0 & 1 & 0 & 0 \\
0 & 0 & \frac{1}{r} & 0 \\
0 & 0 & 0 & \frac{1}{a^{\prime} r \sin \theta}
\end{array}\right) .
$$

The parameter $a^{\prime}$ is associated with the linear mass density $\eta$ of the string by $a^{\prime}=1-4 \eta$. It is defined in the range $(0,1]$ and corresponds to a deficit angle $\Omega=2 \pi\left(1-a^{\prime}\right)$. From the geometrical point of view, the metric in (9) describes a Minkowski space-time with a conical singularity [23].

(ii) In the case of the global monopole with [24-27]

$$
d s^{2}=d t^{2}-d r^{2}-b^{\prime 2} r^{2}\left(d \theta^{2}+\sin ^{2} \theta d \varphi^{2}\right),
$$

we have

$$
\begin{aligned}
& \omega_{\theta a b}=\left(\begin{array}{cccc}
0 & 0 & 0 & 0 \\
0 & 0 & b^{\prime} & 0 \\
0 & -b^{\prime} & 0 & 0 \\
0 & 0 & 0 & 0
\end{array}\right), \\
& \omega_{\varphi a b}=\left(\begin{array}{cccc}
0 & 0 & 0 & 0 \\
0 & 0 & 0 & b^{\prime} \sin \theta \\
0 & 0 & 0 & \cos \theta \\
-b^{\prime} \sin \theta & -\cos \theta & 0 & 0
\end{array}\right),
\end{aligned}
$$

where the tetrad $e_{(a)}^{\mu}$ are

$$
e_{(a)}^{\mu}=\left(\begin{array}{cccc}
1 & 0 & 0 & 0 \\
0 & 1 & 0 & 0 \\
0 & 0 & \frac{1}{b^{\prime} r} & 0 \\
0 & 0 & 0 & \frac{1}{b^{\prime} r \sin \theta}
\end{array}\right) .
$$

The parameter $b^{\prime}$, smaller than unity, depends on the energy scale where the monopole is formed and where the symmetry is broken. The space-time described by (12) has a nonvanishing scalar curvature, $R=2\left(1-b^{\prime}\right) / b^{\prime 2} r^{2}$, and presents a solid angle deficit $\Omega^{\prime}=4 \pi^{2}\left(1-b^{\prime 2}\right)$. The many interesting investigations of physical effects associated with global monopole consider this object as a point-like defect $($ see $[28,29]$ and references therein).

Let us now discuss the problem of Pauli criterion in the presence of $\mathbf{A B}$ potential for both cosmic string and global monopole cases.

2.2. Pauli Criterion and the Aharonov-Bohm Effect: Review. As we know, the KAM satisfy the following relations

$$
\mathbf{L} \times \mathbf{L}=i \hbar \mathbf{L}
$$

with the following standard commutations rules [30]:

$$
\begin{aligned}
& {\left[L_{i}, L_{j}\right]=i \hbar \epsilon_{i j k} L_{k},} \\
& {\left[L_{z}, L_{ \pm}\right]= \pm \hbar L_{ \pm},} \\
& {\left[L^{2}, L_{ \pm}\right]=0}
\end{aligned}
$$

where

$$
L^{2}=-\hbar^{2}\left\{\frac{1}{\sin \theta} \frac{\partial}{\partial \theta}\left(\sin \theta \frac{\partial}{\partial \theta}\right)+\frac{1}{\sin ^{2} \theta} \frac{\partial}{\partial \varphi}\right\},
$$

and where

$$
L_{z}=-i \hbar \frac{\partial}{\partial \varphi}
$$

with $L_{ \pm}=L_{x} \pm i L_{y}$ are the ladder operators. The KAM is based on these commutation relations that Pauli required that the appropriate eigenfunction be those which are square integrable and are closed under the operation of the ladder operators. This condition is called the Pauli criterion, and consequently the commutations relations in (15) are pertinent for the Pauli criterion to be applicable.

In the presence of the $\mathbf{A B}$ potential where

$$
\begin{aligned}
& A_{r}=A_{\theta}=0, \\
& A_{\varphi}=\frac{\Phi}{2 \pi r \sin \theta},
\end{aligned}
$$

the KAM becomes

$$
\begin{aligned}
& L^{2} \\
& =-\hbar^{2}\left\{\frac{1}{\sin \theta} \frac{\partial}{\partial \theta}\left(\sin \theta \frac{\partial}{\partial \theta}\right)+\frac{1}{\sin ^{2} \theta}\left(\frac{\partial}{\partial \varphi}-i \alpha\right)^{2}\right\},
\end{aligned}
$$

with

$$
L_{z}=-i \hbar\left(\frac{\partial}{\partial \varphi}-i \alpha\right)
$$


Here the particle is placed in the vector potential field of an infinitely long solenoid of magnetic flux $\Phi=\alpha \Phi_{0}$ where $\Phi_{0}=$ $\hbar c / e$ and the parameter $\alpha$ is the amount of the magnetic flux.

$\mathrm{Li}[11,12]$ shows that, in the presence of $\mathbf{A B}$ potential, the KAM does not satisfy the fundamental commutation relations (15), and, instead of (15), he obtains

$$
\mathbf{L} \times \mathbf{L}=i \hbar \mathbf{L}+i e \hbar \mathbf{r}(\mathbf{r} \cdot \mathbf{B})
$$

or

$$
\mathbf{L} \times \mathbf{L}=i \hbar \mathbf{L}+2 i \alpha \hbar^{2} \cos \theta \delta\left(\cos ^{2} \theta-1\right) \mathbf{e}_{r},
$$

with the strength of the magnetic field is given by

$$
\mathbf{B}=\frac{\Phi}{\pi r^{2}} \delta\left(\cos ^{2} \theta-1\right) \mathbf{e}_{z}
$$

From (25), both (17) and (18) are transformed into

$$
\begin{aligned}
& {\left[L_{z}, L_{ \pm}\right]= \pm \hbar L_{ \pm},} \\
& {\left[L^{2}, L_{ \pm}\right]} \\
& \quad=\mp 2 \alpha \hbar^{2}\left[L_{ \pm} \delta\left(\cos ^{2} \theta-1\right)+\delta\left(\cos ^{2} \theta-1\right) L_{ \pm}\right] .
\end{aligned}
$$

These rules are different from (15) and (18). As described by $\mathrm{Li}$, the region where the magnetic field exists is inaccessible to the electron; the commutation relations of the KAM should take it into account. This type of commutations relations as said to be global following Li and, consequently, the Pauli criterion is inapplicable.

The eigensolutions of $L_{z}$ and $L^{2}$ under the boundary condition at $\theta=0, \pi$,

$$
\psi(\theta, \varphi)_{\left.\right|_{\theta=o, \pi}}=0,
$$

are presented by Kretzschmar [8]. This condition means that the particle is restricted to the doubly connected region of $\theta \neq$ $0, \pi$. According to Kretzschmar and with the substitutions

$$
\begin{aligned}
L_{z} \psi_{l \lambda}(\theta, \varphi) & =\lambda \hbar \psi_{l \lambda}(\theta, \varphi), \\
L^{2} \psi_{l \lambda}(\theta, \varphi) & =l(l+1) \hbar^{2} \psi_{l \lambda}(\theta, \varphi), \\
\lambda & =m-\alpha, \quad m=(0, \pm 1, \pm 2, \ldots), \\
l & =|\lambda|+\kappa, \quad \kappa=0,1,2, \ldots,
\end{aligned}
$$

the normalized eigenfunctions are of the form

$$
\psi_{\lambda l}(\theta, \varphi)=c_{\lambda, l} P_{l}^{-|\lambda|}(\cos \theta) e^{i m \varphi}, \quad m=0, \pm 1, \pm 2, \ldots,
$$

where

$$
c_{\lambda, l}=e^{(i \pi / 2) \lambda+(i \pi / 2)|\lambda|}\left(\frac{2 l+1}{4 \pi} \frac{\Gamma(l+|\lambda|+1)}{\Gamma(l-|\lambda|+1)}\right)^{1 / 2} .
$$

According to the sign of $\lambda$, we have

$$
\begin{aligned}
& \psi_{\lambda_{1} l_{1}}(\theta, \varphi)=c_{\lambda_{1}, l_{1}} P_{l_{1}}^{-\lambda_{1}}(\cos \theta) e^{i m \varphi}, \\
& \lambda_{1}=m-\alpha>0, l_{1}=\lambda_{1}+\kappa, \\
& \psi_{\lambda_{2} l_{2}}(\theta, \varphi)=c_{\lambda_{2}, l_{2}} P_{l_{2}}^{\lambda_{2}}(\cos \theta) e^{i m \varphi}, \\
& \lambda_{2}=m-\alpha<0, l_{2}=-\lambda_{2}+\kappa .
\end{aligned}
$$

The parameters $\lambda$ and $l$ are dependent on the amount $\alpha$ of the magnetic field which is not correct when we adopt the KAM as written by (1). In addition, the inapplicability of the Pauli criterion modified completely the total Hilbert space $S$. Following Kretzschmar [8], the total Hilbert space $S$ is split into two subspaces, $S_{+}$and $S_{-}$. $S_{+}$is spanned by all the eigenfunctions $\psi_{\lambda_{1} l_{1}}(\theta, \varphi)$, and $S_{-}$is spanned by all the eigenfunctions $\psi_{\lambda_{2} l_{2}}(\theta, \varphi)$. These two subspaces are not connected by the ladder operators.

In this stage, we are able to extend the works of Kretzschmar [8] (splitting of Hilbert space) and Li [11, 12] (the nonapplicability of Pauli's criterion) to the case of scalar bosons in the gravitational field of topological defects such as cosmic string and general monopole: first, we are interested in deducing $\mathbf{L} \times \mathbf{L}$ in the presence of topological defects and then solving the scalar DKP equation by taking into account the works of both authors.

(i) Case of Cosmic String. In cosmic string, replacing the vector $\mathbf{A B}$ by the relation

$$
A_{\varphi}=\frac{\Phi}{2 \pi a^{\prime} r \sin \theta}
$$

(15) is transformed into

$$
\mathbf{L} \times \mathbf{L}=i \hbar \mathbf{L}+\frac{2 i \hbar^{2} \alpha}{a^{\prime}} \cos \theta \delta\left(\cos ^{2} \theta-1\right) \mathbf{e}_{r} .
$$

(ii) Case of Global Monopole. In the global monopole, with

$$
A_{\varphi}=\frac{\Phi}{2 \pi b^{\prime} r \sin \theta}
$$

(15) becomes

$$
\mathbf{L} \times \mathbf{L}=i \hbar \mathbf{L}+\frac{2 i \hbar^{2} \alpha}{b^{\prime 2}} \cos \theta \delta\left(\cos ^{2} \theta-1\right) \mathbf{e}_{r}
$$

Both (34) and (36) are obviously different from (15). According to $\mathrm{Li}[11,12]$, we note that in the presence of $\mathbf{A B}$ potential, the KAM of the particle, in both cases, does not satisfy the fundamental commutation relations of the angular momentum even when the particle is restricted to the doubly connected space where it does not touch the magnetic field on the $z$-axis. The region where the magnetic field exists and is inaccessible to the particle should also be taken into account in the physically meaningful commutation relations. In the limit where $a^{\prime} \rightarrow 1$ or $b^{\prime} \rightarrow 1$, we recover well (25).

In what follows, we are ready to present the solutions of scalar bosons by taking into account that the fundamental commutation relations of the angular momentum are modified in both cosmic string and general monopole cases. 


\section{The DKP Equation with \\ the Aharonov-Bohm and Coulomb \\ Potentials in the Presence of a Cosmic String}

3.1. The Solutions. The cosmic string space-time with an internal magnetic field in spherical coordinates is described by the line element

$$
d s^{2}=d t^{2}-d r^{2}-r^{2} d \theta^{2}-a^{\prime 2} r^{2} \sin ^{2} \theta d \varphi^{2}
$$

The 4-vector potential, in the background of a cosmic string, reads

$$
\begin{aligned}
& A_{0}=\frac{k q}{r}, \\
& A_{r}=0, \\
& A_{\theta}=0, \\
& A_{\varphi}=\frac{\Phi}{2 \pi a^{\prime} r \sin \theta} .
\end{aligned}
$$

From (5), and by using (3), (6), and (10), the DKP equation in the presence of both $\mathbf{A B}$ and Coulomb potentials is given by

$$
\begin{aligned}
& {\left[\frac{\beta^{0}}{\hbar c}\left(E-\frac{k e q}{r}\right)+i \beta^{1} \partial_{r}+\frac{i \beta^{2}}{r}\left(\partial_{\theta}-\beta^{2} \beta^{1}\right)\right.} \\
& +\frac{i \beta^{3}}{a^{\prime} r \sin \theta}\left\{\left(\partial_{\varphi}-i \alpha\right)-a^{\prime} \sin \theta \beta^{3} \beta^{1}\right. \\
& \left.\left.-a^{\prime} \cos \theta \beta^{3} \beta^{2}\right\}-\frac{M c}{\hbar}\right] \psi=0 ;
\end{aligned}
$$

with the wave function, $\psi$ is chosen as

$$
\psi=e^{-i E t / \hbar}\left(\psi_{1}, \psi_{2}, \psi_{3}, \psi_{4}, \psi_{5}\right)^{T}
$$

Inserting (40) into (39) and after an algebraic calculation, we obtain the following system of equations:

$$
\begin{gathered}
\left(\frac{E-k e q / r}{c \hbar}\right) \psi_{2}-i\left(\partial_{r}+\frac{2}{r}\right) \psi_{3} \\
-\frac{i}{r}\left(\partial_{\theta}+\frac{\cos \theta}{\sin \theta}\right) \psi_{4} \\
-\frac{i}{a^{\prime} r \sin \theta}\left(\partial_{\varphi}-i \alpha\right) \psi_{5}=\frac{M c}{\hbar} \psi_{1}, \\
\left(\frac{E-k e q / r}{c \hbar}\right) \psi_{1}=\frac{M c}{\hbar} \psi_{2}, \\
i \partial_{r} \psi_{1}=\frac{M c}{\hbar} \psi_{3}, \\
\frac{i}{r} \partial_{\theta} \psi_{1}=\frac{M c}{\hbar} \psi_{4}, \\
\frac{i}{a^{\prime} r \sin \theta}\left(\partial_{\varphi}-i \alpha\right) \psi_{1}=\frac{M c}{\hbar} \psi_{5} .
\end{gathered}
$$

Putting (42), (43), (44), and (45) into (41), we have

$$
\begin{aligned}
& \left\{\frac{d^{2}}{d r^{2}}+\frac{2}{r} \frac{d}{d r}+\frac{(E-k e q / r)^{2}}{(c \hbar)^{2}}-\left(\frac{M c}{\hbar}\right)^{2}-\frac{L^{2}}{r^{2}}\right\} \psi_{1} \\
& \quad=0
\end{aligned}
$$

where

$$
\begin{aligned}
L^{2} & =-\hbar^{2}\left\{\frac{1}{\sin \theta} \frac{d}{d \theta}\left(\sin \theta \frac{d}{d \theta}\right)\right. \\
& \left.+\frac{1}{a^{\prime 2} \sin ^{2} \theta}\left(\partial_{\varphi}-i \alpha\right)^{2}\right\}, \\
L_{z} & =-i \hbar\left(\frac{d}{d \varphi}-i \alpha\right),
\end{aligned}
$$

and where

$$
\begin{aligned}
& l=|\lambda|+\kappa, \quad(\kappa=0,1,2, \ldots), \\
& \lambda=\frac{m-\alpha}{a^{\prime}},
\end{aligned}
$$

where $l$ are the eigenvalues of $L_{z}$ and $L^{2}$, respectively: we note that (i) these eigenvalues depend on the amount $\alpha$ of the magnetic flux and the geometric parameter of space $a^{\prime}$ and (ii) they are not integer whatever the nature of the parameter $\alpha$.

In order to solve (46), we first put that

$$
\psi_{1}(r, \theta, \phi)=R(r) \chi(\theta, \varphi) .
$$

Expanding (46) leads to

$$
\begin{aligned}
& \left\{\frac{d^{2}}{d r^{2}}+\frac{2}{r} \frac{d}{d r}-\frac{l(l+1)-(k e q / \hbar c)^{2}}{r^{2}}+\frac{E^{2}-M^{2} c^{4}}{\hbar^{2} c^{2}}\right. \\
& \left.-\frac{2 k e q E}{\hbar^{2} c^{2} r}\right\} R(r)=0 .
\end{aligned}
$$

By using the substitutions,

$$
\begin{aligned}
\rho & =\xi r, \\
\xi^{2} & =\frac{4\left(M^{2} c^{4}-E^{2}\right)}{\hbar^{2} c^{2}}, \\
\gamma & =\frac{k e q}{\hbar c}, \\
\varsigma & =\frac{2 \gamma E}{\hbar c \xi},
\end{aligned}
$$

(50) becomes

$$
\begin{aligned}
& \left\{\frac{1}{\rho^{2}} \frac{d}{d \rho}\left(\rho^{2} \frac{d}{d \rho}\right)-\frac{l(l+1)-\gamma^{2}}{\rho^{2}}-\frac{1}{4}-\frac{\varsigma}{\rho}\right\} R(\rho) \\
& \quad=0 .
\end{aligned}
$$


Putting that

$$
R(\rho)=e^{-\rho / 2} \rho^{s} u(\rho),
$$

(52) is transformed into

$$
\begin{aligned}
& \rho \frac{d^{2} u(\rho)}{d \rho^{2}}+\{2(s+1)-\rho\} \frac{d u(\rho)}{d \rho}-(s+\varsigma+1) u(\rho) \\
& \quad=0,
\end{aligned}
$$

with

$$
s(s+1)=l(l+1)-\gamma^{2},
$$

or

$$
s=-\frac{1}{2}+\sqrt{\left(l+\frac{1}{2}\right)^{2}-\gamma^{2}} .
$$

In this stage the solutions are

$$
u(\rho)=N_{\text {norm } 1} F_{1}(s+\varsigma+1 ; 2(s+1), \rho),
$$

with $N_{\text {norm }}$ being the normalization constant and ${ }_{1} F_{1}$ being the confluent series.

The confluent series becomes a polynomial if and only if [30]

$$
s+\varsigma+1=-n, \quad(n=0,1,2, \ldots) .
$$

Consequently, the energy of spectrum is written as

$$
\begin{aligned}
& E_{n} \\
& =\frac{M c^{2}}{\sqrt{1+\gamma^{2} /\left(n+1 / 2+\sqrt{\left(\left|(m-\alpha) / a^{\prime}\right|+\kappa+1 / 2\right)^{2}-\gamma^{2}}\right)^{2}}} .
\end{aligned}
$$

Now, concerning the total wave function and according to the work of [8], we have two cases.

(i) In the subspace $S_{+}$, where $\lambda_{1}>0$ and $l_{1}=\lambda_{1}+\kappa$, the total spinor is

$$
\psi_{n \lambda_{1} l_{1}}\left(\begin{array}{c}
\frac{M c}{\hbar} \\
M c
\end{array} e^{-i E t / \hbar} e^{i m \varphi}\left(\begin{array}{c}
\frac{E-k e q / r}{c \hbar} \\
i \partial_{r} \\
\frac{i}{r} \partial_{\theta} \\
\frac{i}{a^{\prime} r \sin \theta}\left(\partial_{\varphi}-i \alpha\right)
\end{array}\right) \psi_{1}\right. \text {, }
$$

with

$$
\begin{aligned}
\psi_{1}= & N_{\text {norm }} P_{l_{1}}^{-\lambda_{1}}(\cos \theta) \\
& \cdot \rho^{s} e^{-\rho / 2}{ }_{1} F_{1}(s+\varsigma+1 ; 2(s+1), \rho) .
\end{aligned}
$$

(ii) For the subspace $S_{-}$, where $\lambda_{2}<0$ and $l_{2}=-\lambda_{2}+\kappa$, the total spinor is

$\psi_{n \lambda_{2} l_{2}}$

$$
=\frac{\hbar}{M c} e^{-i E t / \hbar} e^{i m \varphi}\left(\begin{array}{c}
\frac{M c}{\hbar} \\
\frac{E-k e q / r}{c \hbar} \\
i \partial_{r} \\
\frac{i}{r} \partial_{\theta} \\
\frac{i}{a^{\prime} r \sin \theta}\left(\partial_{\varphi}-i \alpha\right)
\end{array}\right) \psi_{1},
$$

with

$$
\begin{aligned}
\psi_{1}= & N_{\text {norm }} P_{l_{2}}^{\lambda_{2}}(\cos \theta) \\
& \cdot \rho^{s} e^{-\rho / 2}{ }_{1} F_{1}(s+\varsigma+1 ; 2(s+1), \rho) .
\end{aligned}
$$

\section{The DKP Equation with the Aharonov-Bohm and Coulomb Potentials in the Presence of a Global Monopole}

4.1. The Solutions. The metric of the space-time in the presence of a global monopole is given by [24, 25, 27-29]

$$
d s^{2}=d t^{2}-d r^{2}-b^{\prime 2} r^{2}\left(d \theta^{2}+\sin ^{2} \theta d \varphi^{2}\right) .
$$

By using (3), (6), and (13), the DKP equation with the AB and Coulomb potentials is written as

$$
\begin{aligned}
& {\left[\frac{\beta^{0}}{\hbar c}\left(E-\frac{k e q}{r}\right)+i \beta^{1} \partial_{r}+\frac{i \beta^{2}}{b r}\left(\partial_{\theta}-b^{\prime} \beta^{2} \beta^{1}\right)\right.} \\
& +\frac{i \beta^{3}}{b^{\prime} r \sin \theta}\left\{\left(\partial_{\varphi}-i \alpha\right)-b^{\prime} \sin \theta \beta^{3} \beta^{1}-\cos \theta \beta^{3} \beta^{2}\right\} \\
& \left.-\frac{M c}{\hbar}\right] \Psi=0,
\end{aligned}
$$

with

$$
\Psi=e^{-i(E t / \hbar)}\left(\Psi_{1}, \Psi_{2}, \Psi_{3}, \Psi_{4}, \Psi_{5}\right)^{T},
$$

and with the 4 -vector potential, in the background of a global monopole, reading

$$
\begin{aligned}
& A_{0}=\frac{k q}{r}, \\
& A_{r}=0, \\
& A_{\theta}=0, \\
& A_{\varphi}=\frac{\Phi}{2 \pi b^{\prime} r \sin \theta} .
\end{aligned}
$$


Putting (3) in (65), we obtain the following system of equations:

$$
\begin{aligned}
& \left(\frac{E-k e q / r}{c \hbar}\right) \Psi_{2}-i\left(\partial_{r}+\frac{2}{r}\right) \Psi_{3} \\
& -\frac{i}{b r}\left(\partial_{\theta}+\frac{\cos \theta}{\sin \theta}\right) \Psi_{4} \\
& -\frac{i}{b^{\prime} r \sin \theta}\left(\partial_{\varphi}-i \alpha\right) \Psi_{5}=\frac{M c}{\hbar} \Psi_{1}, \\
& \left(\frac{E-k e q / r}{c \hbar}\right) \Psi_{1}=\frac{M c}{\hbar} \Psi_{2}, \\
& i \partial_{r} \Psi_{1}=\frac{M c}{\hbar} \Psi_{3}, \\
& \frac{i}{b r} \partial_{\theta} \Psi_{1}=\frac{M c}{\hbar} \Psi_{4}, \\
& \frac{i}{b^{\prime} r \sin \theta}\left(\partial_{\varphi}-i \alpha\right) \Psi_{1}=\frac{M c}{\hbar} \Psi_{5} .
\end{aligned}
$$

Inserting, now, (69), (70), (71), and (72) into (68), we obtain

$$
\begin{aligned}
& \left\{\frac{d^{2}}{d r^{2}}+\frac{2}{r} \frac{d}{d r}+\frac{(E-k e q / r)^{2}}{(c \hbar)^{2}}-\left(\frac{M c}{\hbar}\right)^{2}-\frac{L^{2}}{r^{2}}\right\} \Psi_{1} \\
& =0
\end{aligned}
$$

where

$$
\begin{aligned}
& L^{2}=-\hbar^{2}\left\{\frac{1}{\sin \theta} \frac{d}{d \theta}\left(\sin \theta \frac{d}{d \theta}\right)\right. \\
&\left.+\frac{1}{b^{\prime 2} \sin ^{2} \theta}\left(\partial_{\varphi}-i \alpha\right)^{2}\right\}, \\
& L_{z}=-i \hbar\left(\frac{d}{d \varphi}-i \alpha\right), \\
& l=|\lambda|+\kappa, \quad(\kappa=0,1,2, \ldots),
\end{aligned}
$$

with

$$
\lambda=\frac{m-\alpha}{b^{\prime}}
$$

and $l$ are the eigenvalues of $L_{z}$ and $L^{2}$, respectively: we observe that (i) these eigenvalues depend on the amount $\alpha$ of the magnetic flux and the geometric parameter of space $b^{\prime}$ and (ii) they are not integer whatever the nature of the parameter $\alpha$.

In order to solve (73), we first put that

$$
\Psi_{1}(r, \theta, \phi)=R(r) \chi(\theta, \varphi) .
$$

Expanding (73) leads to

$$
\begin{gathered}
\left\{\frac{d^{2}}{d r^{2}}+\frac{2}{r} \frac{d}{d r}+\frac{1}{(c \hbar)^{2}}\left(E-\frac{k e q}{r}\right)^{2}-\left(\frac{M c}{\hbar}\right)^{2}\right. \\
\left.-\frac{l(l+1) / b^{\prime 2}}{r^{2}}\right\} \Psi_{1}(r)=0 .
\end{gathered}
$$

Using the same change of variables as in the case of cosmic string, (77) is transformed into

$$
\frac{d^{2} R(r)}{d \rho^{2}}+\left(-\frac{l(l+1) / b^{\prime 2}-\gamma^{2}}{\rho^{2}}-\frac{\zeta}{\rho}-\frac{1}{4}\right) R(r)=0 .
$$

Now let us make a change of variable

$$
R(r)=\rho^{a} e^{-\rho / 2} H(\rho),
$$

and, putting it in (78), we have

$$
\begin{gathered}
\rho^{2} \frac{d^{2} H(\rho)}{d \rho^{2}}+\left\{2(a+1) \rho-\rho^{2}\right\} \frac{d H(\rho)}{d \rho} \\
+\left[\left\{a(a+1)-\left(\frac{l(l+1)}{b^{\prime 2}}-\gamma^{2}\right)\right\}\right. \\
-(\zeta+a+1) \rho] H(\rho)=0,
\end{gathered}
$$

with

$$
a=-\frac{1}{2} \pm\left\{\frac{l(l+1)}{b^{\prime 2}}+\frac{1}{4}-\gamma^{2}\right\}^{1 / 2}
$$

To solve (80), we use the Frobenius method [31-34]. This can be written as a power series expansion around the origin:

$$
H(\rho)=\sum_{k=0}^{\infty} c_{k} \rho^{k}
$$

Inserting (82) into (80), we obtain the following recurrence relation:

$$
c_{k+1}=\frac{k+(\zeta+a+1)}{k(k+1)+2(a+1)(k+1)} c_{k} .
$$

Now, when $k \rightarrow \infty$, the ration $c_{k+1} / c_{k} \rightarrow 0$ : we can understand this condition by saying that special kind of exact solutions, which represent bound states, can be obtained looking for polynomials expressions to $H(\rho)$; that is, the solutions can be obtained by imposing the conditions where power series becomes a polynomial of degree $n$. This can be argued as follows: in quantum mechanics, if we want to have a normalizable wave function, we have to impose that $R(\rho)$ vanishes at $\rho \rightarrow 0$ and $\rho \rightarrow \infty$. In this way, bound state solutions can be obtained because there is no divergence of the wave function at $\rho \rightarrow 0$ and $\rho \rightarrow \infty$. In our case, $H(\rho)$ has written as a power series expansion around the origin (see (82)). As a result of that, the solutions can be achieved by imposing that the power series expansion (82) or the biconfluent Heun series becomes a polynomial of degree $n$. This guaranteed that $R(\rho)$ becomes $F$ as $\rho$ at the origin and vanishes at $\rho \rightarrow \infty$ [35-40]. Thus, in order that the power series expansion becomes a polynomial of degree $n$, we impose that

$$
\zeta+a+1=-n
$$


In this case, the form of spectrum of energy is

$$
\begin{aligned}
E_{n} & =M c^{2}\{1 \\
& \left.+\frac{\gamma^{2}}{\left[n+1 / 2+\left\{l(l+1) / b^{2}+1 / 4-\gamma^{2}\right\}^{1 / 2}\right]^{2}}\right\}^{-1 / 2} .
\end{aligned}
$$

Now, concerning the total wave function, we have also two cases [8].

(i) In the subspace $S_{+}$, where $\lambda_{1}>0$ and $l_{1}=\lambda_{1}+\kappa$, the total spinor is

$$
\begin{aligned}
& \Psi_{n \lambda_{1} l_{1}} \\
& =\frac{\hbar}{M c} e^{-i E t / \hbar} e^{i m \varphi}\left(\begin{array}{c}
\frac{M c}{\hbar} \\
\frac{E-k e q / r}{c \hbar} \\
i \partial_{r} \\
\frac{i}{r} \partial_{\theta} \\
\frac{i}{b^{\prime} r \sin \theta}\left(\partial_{\varphi}-i m_{0}\right)
\end{array}\right) \psi_{1},
\end{aligned}
$$

with

$$
\begin{aligned}
\psi_{1}= & N_{\text {norm }}^{\prime} P_{l_{1}}^{-\lambda_{1}}(\cos \theta) \\
& \cdot \rho^{a} e^{-\rho / 2}{ }_{1} F_{1}(a+\varsigma+1 ; 2(a+1), \rho) .
\end{aligned}
$$

(ii) For the subspace $S_{-}$, where $\lambda_{2}<0$ and $l_{2}=-\lambda_{2}+\kappa$, the total spinor is

$$
=\frac{\hbar}{M c} e^{-i E t / \hbar} e^{i m \varphi}\left(\begin{array}{c}
\frac{M c}{\hbar} \\
\frac{E-k e q / r}{c \hbar} \\
i \partial_{r} \\
\frac{i}{r} \partial_{\theta} \\
\frac{i}{b^{\prime} r \sin \theta}\left(\partial_{\varphi}-i \alpha\right)
\end{array}\right) \psi_{1} \text {, }
$$

with

$$
\begin{aligned}
\psi_{1}= & N_{\text {norm }}^{\prime} P_{l_{2}}^{\lambda_{2}}(\cos \theta) \\
& \cdot \rho^{a} e^{-\rho / 2}{ }_{1} F_{1}(a+\varsigma+1 ; 2(a+1), \rho) .
\end{aligned}
$$

Here, $N_{\text {norm }}^{\prime}$ is the normalization constant and ${ }_{1} F_{1}$ is the confluent series.

\section{Conclusion}

This paper is devoted to studying the solutions of the relativistic quantum motion of a charged scalar particles in the presence of an Aharonov-Bohm and Coulomb potentials in the space-times produced by an idealized cosmic string and global monopole. These solutions have been obtained, and the influence of the parameter of the geometry of both spaces has been discussed. In addition, the remarks which $\mathrm{Li}[11,12]$ has been proposed concerning the $\mathbf{A B}$ effect have been extended in our case: thus, the presence of $\mathbf{A B}$ potential changes completely the fundamental commutation relations of the angular momentum. Following the works of $[11,12]$, we note that (i) the KAM are not satisfied even when the particle is restricted to the doubly connected space where it does not touch the magnetic field on the $z$-axis, (ii) the region where the magnetic field exists and is inaccessible to the electron should be taken into account in the physical commutation relations, and finally (iii) the Pauli criterion which said that "the appropriate eigenfunctions are those which are square integrable and are closed under the operation of ladder operators" is inapplicable to the vector AB. The existence of the magnetic field on the $z$-axis is the principal cause of breaking down the symmetry of particle's motion around the $z$-axis.

The eigenfunctions and eigenvalues of $L_{z}$ and $L^{2}$ have been presented under the following boundary condition: $\left.\psi(r, \theta, \varphi)\right|_{\theta=0, \pi}=0$, and the space $S$, as shown by [8], is split into two subspaces, $S_{+}$and $S_{-}: S_{+}$is spanned by all the wave function $\psi_{n l_{1} \lambda_{1}}(\theta, \varphi)$ for cosmic string $\left(\Psi_{n l_{1} \lambda_{1}}(\theta, \varphi)\right.$ for global monopole) and $S_{-}$is spanned by all the wave function $\psi_{n l_{2} \lambda_{2}}(\theta, \varphi)$ for cosmic string $\left(\Psi_{n l_{2} \lambda_{2}}(\theta, \varphi)\right.$ for global monopole). The eigensolutions in the gravitational field of cosmic string and a global monopole (i) are different (ii) and depend on the geometry of spaces.

\section{Conflicts of Interest}

The authors declare that they have no conflicts of interest.

\section{References}

[1] G. Petiau, Mémoires de la Classe des Sciences Collection, vol. 16, no 2, University of paris thesis, R. Belg., Mém. Cl. Sci. Académie Royale de Belgique, 1936.

[2] R. J. Duffin, "On the characteristic matrices of covariant systems," Physical Review A: Atomic, Molecular and Optical Physics, vol. 54, no. 12, p. 1114, 1938.

[3] N. Kemmer, "Quantum theory of Einstein-Bose particles and nuclear interaction," Proceedings of the Royal Society of London, vol. A166, no. 127, 1938.

[4] N. Kemmer, "The particle aspect of meson theory," Proceedings of the Royal Society of London, vol. A173, no. 91, 1939.

[5] A. L. Cavalcanti de Oliveira and E. R. Bezerra de Mello, "Exact solutions of the Klein-Gordon equation in the presence of a dyon, magnetic flux and scalar potential in the spacetime of gravitational defects," Classical and Quantum Gravity, vol. 23, no. 17, pp. 5249-5263, 2006. 
[6] Y. Aharonov and D. Bohm, "Significance of electromagnetic potentials in the quantum theory," Physical Review A: Atomic, Molecular and Optical Physics, vol. 115, no. 3, article 485, 1959.

[7] M. Socolovsky, Aharonov-Bohm Effect, vol. 191, Elsevier Ltd, 2006.

[8] M. Kretzschmar, "On the Aharonov-Bohm effect for bound states," Zeitschrift für Physik, vol. 185, no. 2, pp. 97-110, 1965.

[9] W. C. Henneberger, "Sturm-Liouville eigenproblems with an interior pole," Journal of Mathematical Physics, vol. 22, no. 8, pp. 1575-1590, 1981.

[10] S. M. Roy and V. Singh, "Time-dependent Aharonov-Bohm Hamiltonian and admissibility criteria of quantum wave functions," Il Nuovo Cimento, vol. 79, pp. 391-409, 1984.

[11] C.-F. Li, "Pauli criterion and the vector Aharonov-Bohm effect," Annals of Physics, vol. 252, no. 2, pp. 329-335, 1996.

[12] C.-F. Li, "Gauge transformation and A-B effect," Physica B: Condensed Matter, vol. 229, no. 3-4, pp. 354-360, 1997.

[13] A. Boumali, "Zero spin particle in an Aharonov-Bohm potential," Canadian Journal of Physics, vol. 82, no. 1, pp. 67-74, 2004.

[14] Y. Nedjadi and R. C. Barrett, "The Duffin-Kemmer-Petiau oscillator," Journal of Physics A: Mathematical and General, vol. 27, no. 12, pp. 4301-4315, 1994.

[15] M. Falek and M. Merad, "Duffin-Kemmer-Petiau equation in Robertson-Walker space-time," Central European Journal of Physics, vol. 8, no. 3, pp. 408-414, 2010.

[16] K. Sogut and A. Havare, "Spin-1 particle in an electrical field in $(1+1)$-dimensional Schrödinger spacetime," Classical and Quantum Gravity, vol. 23, no. 23, pp. 7129-7142, 2006.

[17] J. T. Lunardi, B. M. Pimentel, and R. G. Teixeira, "DuffinKemmer-Petiau equation in Riemannian space-times," Geometrical Aspects of Quantum Fields, p. 111, 2001, https://arxiv.org/ abs/gr-qc/9909033.

[18] M. Nakahara, Geometry, Topology and Physics, IOP Publishing Ltd, 2003.

[19] G. D. A. Marques and V. B. Bezerra, "Non-relativistic quantum systems on topological defects spacetimes," Classical and Quantum Gravity, vol. 19, no. 5, p. 985, 2002.

[20] R. Kalita and D. Borah, "Discriminating Majorana neutrino phases in the light of lepton flavor violation and leptogenesis in type I+II seesaw models," International Journal of Modern Physics A, vol. 30, no. 22, Article ID 1550130, 24 pages, 2015.

[21] G. D. A. Marques, J. G. De Assis, and V. B. Bezerra, "Some effects on quantum systems due to the gravitational field of a cosmic string," Journal of Mathematical Physics, vol. 48, no. 11, Article ID 112501, 2007.

[22] Z. Wang, Z. Long, C. Long, and J. Teng, "Exact solutions of the Schrödinger equation with a coulomb ring-shaped potential in the cosmic string spacetime," Physica Scripta, vol. 90, Article ID 055201, 2015.

[23] M. Hosseinpour, F. M. Andrade, E. O. Silva, and H. Hassanabadi, "Erratum to: Scattering and bound states for the Hulthén potential in a cosmic string background," The European Physical Journal C, vol. 77, no. 270, 2017.

[24] M. Barriola and A. Vilenkin, "Gravitational field of a global monopole," Physical Review Letters, vol. 63, no. 4, pp. 341-343, 1989.

[25] G. D. A. Marques and V. B. Bezerra, "Hydrogen atom in the gravitational fields of topological defects," Physical Review D: Particles, Fields, Gravitation and Cosmology, vol. 66, no. 10, Article ID 105011, 2002.
[26] E. R. Bezerra de Mello and A. A. Saharian, "Polarization of the fermionic vacuum by a global monopole with finite core," Physical Review D, vol. 75, Article ID 065019, 2007.

[27] E. R. B. de Mello and A. A. Saharian, "Scalar self-energy for a charged particle in global monopole spacetime with a spherical boundary," Classical and Quantum Gravity, vol. 29, no. 13, Article ID 135007, p. 25, 2012.

[28] E. R. Bezerra de Mello and C. Furtado, "Nonrelativistic scattering problem by a global monopole," Physical Review D: Particles, Fields, Gravitation and Cosmology, vol. 56, no. 2, pp. 1345-1348, 1997.

[29] D. Barbosa, U. de Freitas, and E. R. Bezerra de Mello, "Induced self-energy on a static scalar charged particle in the spacetime of a global monopole with finite core," Classical and Quantum Gravity, vol. 28, no. 6, 065009, 23 pages, 2011.

[30] A. S. Davydov, Quantum Mechanics, Pergamon Press, New York, NY, USA, 1976.

[31] S. Y. Slavyanov and W. Lay, Special Functions: A Unified Theory Based on Singularities, Oxford University Press, Oxford, UK, 2000.

[32] G. B. Arfken and H. J. Weber, Mathematical Methods for Physists, Elsevier Academic Press, New York, NY, USA, 6th edition, 2005.

[33] P. Marioni, "Sur la forme bi-confluente de l'equation de Heun," Comptes Rendus de l'académie des Sciences Numérisés, vol. 264, pp. 503-505, 1967.

[34] A. Ronveaux, Heun's Differential Equations, Oxford University Press, New York, NY, USA, 1995.

[35] K. Bakke and F. Moraes, "Threading dislocation densities in semiconductor crystals: a geometric approach," Physics Letters A, vol. 376, no. 45, pp. 2838-2841, 2012.

[36] K. Bakke, "Bound states for a Coulomb-type potential induced by the interaction between a moving electric quadrupole moment and a magneticfield," Annals of Physics, vol. 341, pp. 86-93, 2014.

[37] I. C. Fonseca and K. Bakke, "Rotating effects on an atom with a magnetic quadrupole moment confined to a quantum ring," The European Physical Journal Plus, vol. 131, no. 67, 2016.

[38] E. R. Figueiredo Medeiros and E. R. Bezerra de Mello, "Relativistic quantum dynamics of a charged particle in cosmic string spacetime in the presence of magnetic field and scalar potential," The European Physical Journal C, vol. 72, article 2051, 2012.

[39] K. Bakke and C. Furtado, "On the Klein-Gordon oscillator subject to a Coulomb-type potential," Annals of Physics, vol. 355, pp. 48-54, 2015.

[40] G. D. A. Marques, V. B. Bezerra, and S. G. Fernandes, "Exact solution of the dirac equation for a coulomb and scalar potentials in the gravitational field of a cosmic string," Physics Letters A, vol. 341, no. 1-4, pp. 39-47, 2005. 

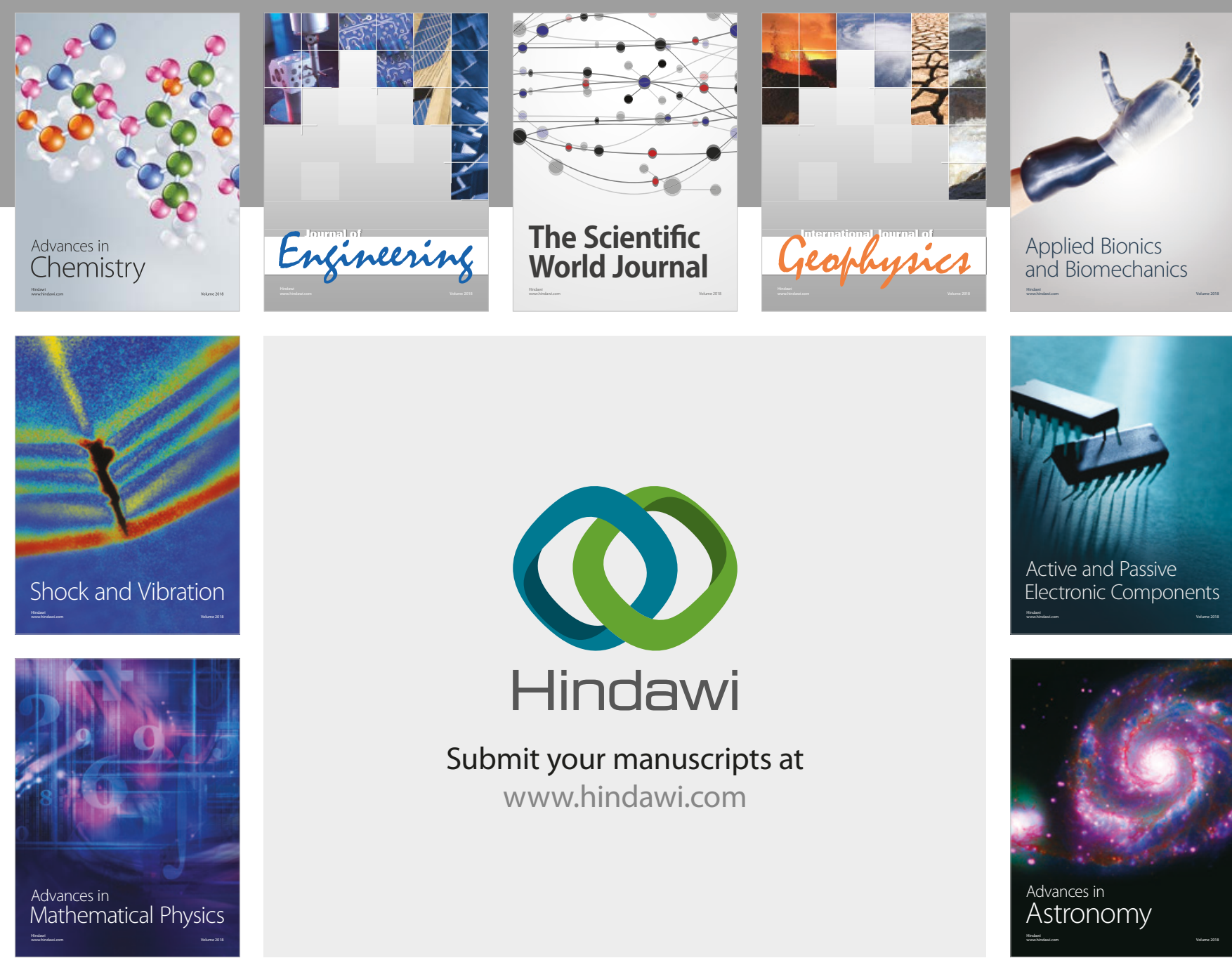

Submit your manuscripts at

www.hindawi.com

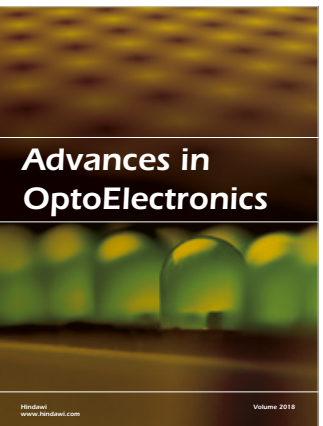

\section{Rotcting Machinery}
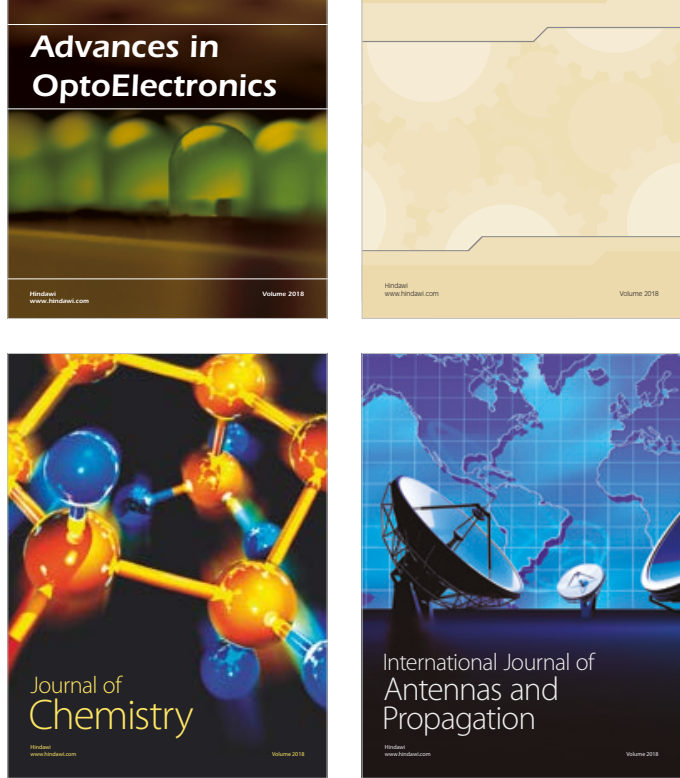

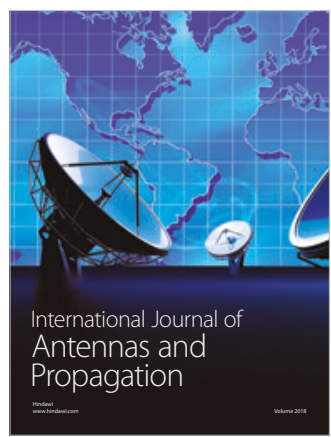

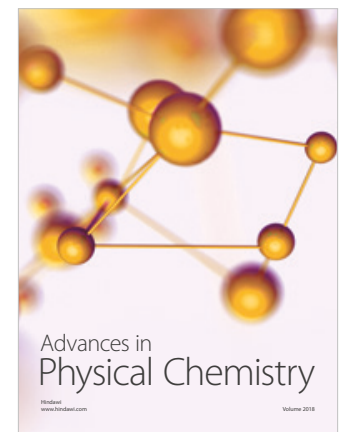

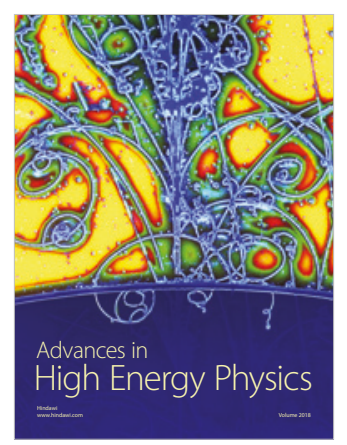

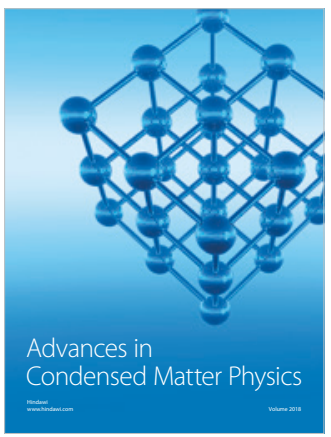

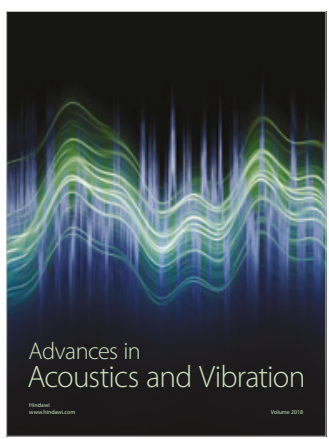

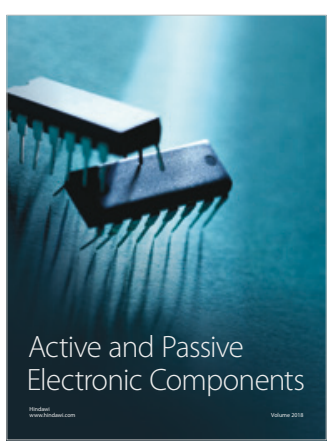
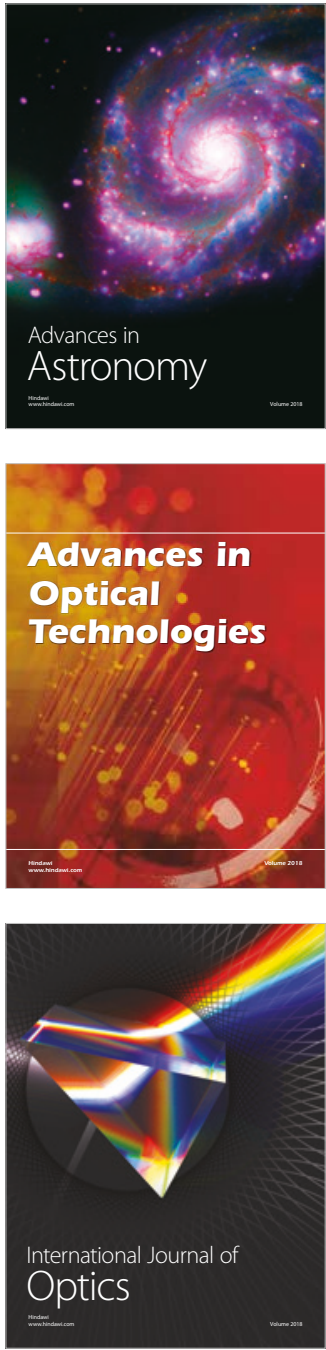